\title{
The Role of Objectification in the Victimization and Perpetration of Intimate Partner Violence
}

\author{
Melissa R. Jonnson, BA \\ Jennifer I. Langille, MA \\ Zach Walsh, PhD \\ University of British Columbia, Kelowna, Canada
}

\begin{abstract}
Intimate partner violence (IPV) is a substantial health concern and identifying risk factors for IPV is a research priority. We examined the relationship between severe IPV and objectification of the self and other sex across participant sex. A sample of 1,005 male and female university students completed a series of online questionnaires that measure levels of self-objectification, objectification of the other sex, and histories of severe IPV victimization and perpetration. Self-objectification was associated with severe psychological aggression, physical assault, and sexual coercion victimization in females, but not in males. Objectification of the other sex was associated with severe psychological aggression and physical assault perpetration in males, but not in females. These findings contribute to our understanding of gender similarities and differences in IPV.
\end{abstract}

Keywords: psychological aggression; physical assault; sexual coercion; objectification theory; gender

I ntimate partner violence (IPV) is a pervasive health concern that is associated with substantial costs to individuals and societies (Centers for Disease Control and Prevention [CDC], 2010). It accounts for a quarter of all violent crimes reported to police, with the highest rates experienced among young adults (Statistics Canada, 2011). Indeed, approximately $30 \%$ of university students report engaging in IPV (Fass, Benson, \& Leggett, 2008; Straus, 2004). Although violence in relationships is often bidirectional, women are overrepresented as primary victims and underrepresented as primary perpetrators (Graham-Kevan \& Archer, 2003; Hamby, 2016; Henning, Renauer, \& Holdford, 2006; Melton \& Sillito, 2012; Walsh et al., 2010; World Health Organization [WHO], 2012). In addition, women generally sustain more severe injuries from IPV (Straus, 2004; Tjaden \& Thoennes, 2000). These findings have evoked a call for continued research into gendered similarities and differences in IPV experiences (Ansara \& Hindin, 2010; Johnson, 2006; Straus, 2006). Identifying correlates of IPV perpetration and victimization may help to improve prevention and intervention strategies.

Evidence suggests that IPV is best explained by a multiple-causal model that considers a combination of individual, relational, societal, and community factors (CDC, 2010; WHO, 2012). The objectification of women, which occurs at individual, relational, and 
societal levels, has been linked to IPV perpetration and victimization (Davidson \& Gervais, 2015; Gervais, DiLillo, \& McChargue, 2014; Hald, Malamuth, \& Yuen, 2010; Tully, 2012). Objectification is defined as "the experience of being treated as a body (or collection of body parts) valued predominantly for its use to (or consumption by) others" (Fredrickson \& Roberts, 1997, p. 174). According to objectification theory, frequent and chronic exposure to objectification can facilitate the internalization of an objectified perspective of oneself, in which one's social worth is reduced to one's appearance (Fredrickson \& Roberts, 1997). Because of the widespread sexual objectification of women in society, women and girls are particularly likely to be the targets of interpersonal objectification and, further, to internalize an objectified perception of themselves (Calogero, 2011; Davidson \& Gervais, 2015; Ward, Vandenbosch, \& Eggermont, 2015). Self-objectification has been associated with a range of clinically problematic conditions including body shame, poor self-esteem, sexual dysfunction, and an increased risk for anxiety, depression, eating disorders, and violence (Calogero, 2011; Gervais \& Davidson, 2013; Mercurio \& Landry, 2008; Riva, Gaudio, \& Dakanalis, 2015).

\section{OBJECTIFICATION AND GENDER}

Although most research on objectification has investigated its effects on women, recent research suggests that men are also subject to and meaningfully affected by objectification (Davidson, Gervais, Canivez, \& Cole, 2013; Green et al., 2014; Loughnan \& Pacilli, 2014). The applicability of objectification theory to men is currently under exploration; however, researchers have emphasized the importance of evaluating cross-gender consistency in the construct of objectification (Heimerdinger-Edwards, Vogel, \& Hammer, 2011; Moradi \& Huang, 2008). Previous work has discovered differences in men's and women's relationships with objectification (Aubrey, Hopper, \& Mbure, 2011; Strelan \& Hargreaves, 2005). Specifically, research indicates that women, as a group, are objectified to a greater degree than men by both genders. Men objectify women, however, to a greater degree than women objectify other women. In addition, women generally self-objectify to a greater extent than men self-objectify (Strelan \& Hargreaves, 2005). Differences in self-objectification are evident by preadolescence (Lindberg, Hyde, \& McKinley, 2006). Although levels of self- and other-objectification vary across genders, many of the adverse effects of self-objectification (e.g., body shame, depression, and disordered eating) appear to be similar for men and women (Dakanalis, Timko, Clerici, Zanetti, \& Riva, 2014; Green et al., 2014; Martins, Tiggemann, \& Kirkbride, 2007; Moradi \& Huang, 2008; Riva et al., 2015; Wiseman \& Moradi, 2010).

\section{OBJECTIFICATION AND ATTITUDES TOWARD INTIMATE PARTNER VIOLENCE}

In the context of IPV, research reveals that media portrayals of women can influence people's attitudes toward interpersonal violence and rape (Aubrey et al., 2011; Lanis \& Covell, 1995). For instance, Lanis and Covell (1995) randomly assigned a sample of male and female university students to evaluate one of three types of advertisements: those including sexually objectifying depictions of women, those including progressive depictions of women, or those including the product only. Males who were exposed to ads with sexually objectifying portrayals of women were more accepting of interpersonal violence and rape myths than males who were exposed to nonobjectifying stimuli. In contrast, 
females who were exposed to ads with liberal depictions of women were less likely to endorse such attitudes than females exposed to nonprogressive stimuli. Correlational research has also discovered that males who score high in trait objectification hold more supportive attitudes toward IPV than males who score low in trait objectification (Tully, 2012). In particular, they assign less responsibility to perpetrators, are less sympathetic toward victims, and perceive victims to be less harmed (Tully, 2012).

\section{OBJECTIFICATION AND INTIMATE PARTNER VIOLENCE PERPETRATION AND VICTIMIZATION}

Beyond affecting attitudes toward interpersonal and sexual violence, objectification has been linked to IPV perpetration and victimization. For example, objectification of women has been identified as a moderating theme in male batterer interviews, as well as in $70 \%$ of male sex offender interviews (Polaschek \& Gannon, 2004; Tilley \& Brackley, 2005). Research has also discovered a direct association between IPV victimization and selfobjectification. Davidson and Gervais (2015) found that women with a history of IPV victimization were more likely to experience self-objectification, body shame, and body surveillance than those without a history. Furthermore, the relationship between IPV and body shame were significantly mediated by the combined effect of self-objectification and body surveillance. Taken together, these findings suggest that objectification may be an important component to understanding the perpetration and victimization of IPV.

\section{STUDY GOALS AND HYPOTHESES}

To date, research regarding objectification and IPV has focused predominantly on the objectification of women by males with a history of IPV perpetration. To our knowledge, research has not yet examined objectification of males by females with a history of IPV perpetration, and only limited research has assessed the link between self-objectification and IPV victimization. In addition, prior studies have not distinguished between minor and severe violence, which evidence indicates may differ conceptually and etiologically (Straus, 2008). This study adds to prior literature by examining whether objectification of the self or members of the other sex is related to the frequency of severe IPV perpetration or victimization. Furthermore, this is the first study to use a standardized measure of objectification to assess objectification of the other sex in people with a history of IPV perpetration.

This study distinguishes between primarily unidirectional IPV and bidirectional IPV. Based on prior research of gender differences in objectification tendencies, we expect that objectification of the self will be exacerbated in females with a history of severe, primarily unidirectional IPV victimization and objectification of the other sex will be exacerbated in males with a history of severe, primarily unidirectional IPV perpetration. Given that people with a history of bidirectional IPV have both perpetrated and been victimized by severe IPV, we make no predictions about their levels of self- or other-objectification. Accordingly, the study hypotheses are as follows:

1. Levels of self-objectification will be higher among females with a history of severe, primarily unidirectional IPV victimization compared to those with no history of IPV.

2. Levels of objectification of the other sex will be higher among males with a history of severe, primarily unidirectional IPV perpetration compared to those with no history of IPV. 


\section{METHOD}

\section{Participants}

The sample consisted of 1,005 female $(70.8 \%)$ and male $(29.2 \%)$ students recruited through an online research participant pool at a Canadian university. Participants were required to have been in a relationship with a member of the other sex within the past year. The sample ranged in age from 17 to 45 years old $\left(M_{\text {age }}=20\right.$ years, $\left.S D=2.70\right)$. The majority of participants identified as White (65\%), $15.3 \%$ as Asian, $9.2 \%$ as Middle Eastern or South Asian, and $10.5 \%$ identified as other groups. Regarding education level, $28.3 \%$ were first year students, $29.6 \%$ second year, $19.9 \%$ third year, $17.8 \%$ fourth year, and $4.4 \%$ were beyond their fourth year of education. Participants provided informed consent and received their choice of course credit or $\$ 20$ as compensation for their participation. Research Ethics Board approval was obtained for this research.

\section{Materials and Procedure}

Objectification Questionnaires. Self-objectification was measured with the SelfObjectification Questionnaire (SOQ; Noll \& Fredrickson, 1998), in which participants are given a list of 10 attributes and are asked to rank them in order of importance to their self-concept. Five of these attributes are observable, appearance-based traits (e.g., physical attractiveness), whereas the other five are nonobservable, competence-based traits (e.g., health). The sum of the appearance ratings is subtracted from the sum of the competence ratings and the difference is used as an indicator of self-objectification. Scores range from -25 to 25 , with higher scores representing higher levels of self-objectification. An issue that has been identified in previous research is that participants may misunderstand instructions for completing the SOQ and assign the same rank to multiple attributes (Calogero, 2011). To address this issue, participants had to "drag and drop" attributes into their preferred order, eliminating the possibility of more than one attribute sharing the same rank.

Objectification of the other sex was measured with a modified version of the SOQ, in which participants were asked to rank the importance of these same attributes in the other sex. In this case, higher scores represent greater objectification of the other sex. For this study, we refer to this measure as the Objectification of the Other Sex Questionnaire (OOSQ). Prior studies have made a similar adaptation to the SOQ to measure people's objectification of others of the same and other gender, termed the Other-Objectification Questionnaire (OOQ; Davidson, Gervais, \& Sherd, 2015; Strelan \& Hargreaves, 2005).

The SOQ is grounded in objectification theory (Fredrickson \& Roberts, 1997), which posits that self-objectification arises from concern with one's physical appearance irrespective of people's satisfaction with or valuation of their appearance (Noll \& Fredrickson, 1998). As such, objectification can be internalized by individuals who are satisfied with their bodies, as well as by those who are dissatisfied. The SOQ demonstrates satisfactory construct, convergent, and discriminant validity (Calogero, 2011; Noll \& Fredrickson, 1998). Specifically, SOQ scores are positively correlated with theoretically related constructs such as appearance anxiety $(r=.52, p<.01)$ and body size dissatisfaction $(r=.46, p<.01$; Noll \& Fredrickson, 1998); SOQ scores are unrelated to body mass index $(r=-.02$, Fredrickson, Roberts, Noll, Quinn, \& Twenge, 1998; $r=-.10$, Tiggemann \& Lynch, 2001), which distinguishes self-objectification from body size as a unique component of people's physical self concepts. 
Given that the SOQ is an ipsative measure that produces rank-ordered data, traditional methods for inspecting its internal consistency reliability are not suitable. Instead, past research has provided the correlation between the appearance-based and competencebased traits as an index of item interrelation (Davidson et al., 2015; Hill \& Fischer, 2008). Hill and Fischer (2008) reasoned that the sum of the appearance-based and sum of the competence-based traits should be negatively correlated because people who rank appearance-based attributes as more important should conversely rank competence-based attributes as less important. With respect to the SOQ, Hill and Fischer (2008) identified a negative correlation between appearance-based and competence-based traits $(r=-.81$, $p<.001$ ) and, with respect to the OOQ, Davidson et al. (2015) identified a negative correlation between these two sets of traits $(r=-.98, p<.001$ for women; $r=-.97, p<.001$ for men). Using the same method, this study also found a negative correlation between appearance-based and competence-based traits for both the SOQ $(r=-1.00, p<.001)$ and the OOSQ $(r=-1.00, p<.001)$. The perfect correlation identified in this study can be explained by the fact that the sums of the appearance-based and competence-based traits are linearly dependent. The reason that the previously mentioned studies did not find a perfect correlation could be that their participants were able to inadvertently assign the same rank to multiple traits, whereas the novel drag and drop technique designed for this study prevents this measurement error from occurring.

Histories of Intimate Partner Violence. Histories of psychological, physical, and sexual IPV were measured using the Revised Conflict Tactics Scale (CTS2; Straus, Hamby, Boney-McCoy, \& Sugarman, 1996), which assesses both the type and frequency of violent behavior by both partners within the past year. The CTS2 consists of 39 item pairs which can be divided into five subscales: Negotiation (6 item pairs; e.g., "I showed my partner I cared even though we disagreed"), Physical Assault (12 item pairs; e.g., "I beat up my partner"), Psychological Aggression (8 item pairs; e.g., "I called my partner fat or ugly"), Sexual Coercion (7 item pairs; e.g., "I used threats to make my partner have sex"), and Injury (6 item pairs; e.g., "I had a broken bone from a fight with my partner"). Items are also classified into minor and severe acts of violence, with severe acts involving a higher potential for injury (e.g., "I used a knife or gun on my partner") than minor acts (e.g., "I pushed or shoved my partner"). Participants report how many times they have employed or experienced each act in the past year, with response options ranging from 0 (never) to 6 (more than 20 times). Prevalence and chronicity scores were computed for each of the CTS2 subscales following standard scoring procedures (Straus et al., 1996). Minor and severe violence was analyzed separately.

Regarding occurrence, previous studies using the CTS2 indicate that $17 \%-47 \%$ of college students have physically assaulted an intimate partner within the past year (Straus, 2004). Prior research indicates that the CTS2 may overestimate the frequency of violent behaviors in young, dating, nonclinical samples because of some participants reporting playful or mock violence as meaningful violence (Hamby, 2016; Lehrner \& Allen, 2014). The majority of our sample $(81.0 \%)$ reported committing or experiencing at least one act of minor IPV (psychological, physical, or sexual) within the past year, suggesting an oversensitivity in minor IPV detection. Research supports the importance of screening out minor incidents of IPV in populations that are more extensively involved in relationship violence to increase accuracy and provide information that is most relevant to practitioners, policymakers, and law enforcement (Hamby \& Turner, 2013). Accordingly, to avoid miscategorizing nonviolent participants as positive for IPV history, only the severe CTS2 subscales were used for assessing participants' histories of IPV. The prevalence of severe 
IPV (psychological, physical, and sexual) in our sample was within the range detected in previous studies, with $34.8 \%$ of participants reporting experiences of severe IPV victimization $(9.3 \%)$, severe IPV perpetration (11\%), or both $(14.5 \%)$.

In this study, a chronicity score of greater than or equal to one $(\geq 1)$ on the severe psychological aggression, severe physical assault, or severe sexual coercion subscale was classified as positive for IPV history. People with a history of primary IPV perpetration $(n=111)$ consisted of those who reported perpetrating one or more severe incidents of violence against their partners and who either had no history of severe IPV victimization or who reported perpetrating severe IPV at least twice as frequently as they reported severe IPV victimization. People with a history of primary IPV victimization $(n=93)$ consisted of those who reported severe IPV victimization one or more times by their partners and who either reported no history of severe IPV perpetration or who reported severe victimization at least twice as frequently as they reported severe perpetration. People engaged in bidirectional violence $(n=146)$ consisted of those who reported approximately equivalent rates of severe IPV perpetration and victimization (i.e., where neither rate exceeded twice that of the other). People in the nonviolent comparison group $(n=655)$ consisted of those who had no history of either severe IPV perpetration or victimization.

The CTS2 is the most commonly used self-report measure of IPV and is empirically supported for use with university students (Straus et al., 1996). Internal consistency reliability coefficients from the development of the scale include .79 (Psychological Aggression), .86 (Physical Assault), and .87 (Sexual Coercion; Straus et al., 1996). Consistent with these estimates, the internal consistency reliability coefficients obtained for this study were .70 (Psychological Aggression), .89 (Physical Assault), and .71 (Sexual Coercion). Concurrent validity was assessed by analyzing the correlations between IPV perpetration subscales that should theoretically be associated: Psychological Aggression and Physical Assault $(r=.74, p<.001)$, Psychological Aggression and Sexual Coercion $(r=.75, p<.001)$, and Physical Assault and Sexual Coercion $(r=.88, p<.001)$. Discriminant validity was assessed by analyzing the correlations between IPV perpetration subscales that should theoretically be distinct: Negotiation and Psychological Aggression $(r=.03, p=.38)$, Negotiation and Physical Assault $(r=-.04, p=.18)$, and Negotiation and Sexual Coercion $(r=-.05, p=.14)$. Past research provides evidence of strong test-retest reliability, with correlations in the range of $r=.67-.72$ for the IPV perpetration subscales and $r=.65-.86$ for the IPV victimization subscales (Vega \& O'Leary, 2007).

It is important to note that there has been considerable disagreement regarding the meaning of CTS2 scores, especially in cases of female-perpetrated and bidirectional IPV (Renner, Reese, Peek-Asa, \& Ramirez, 2015; Woodin, Sotskova, \& O’Leary, 2013). Although a review of this controversy is beyond the scope of the current article, there are several pertinent articles that interested readers can peruse (e.g., Archer, 2000; Frieze, 2000; O’Leary, 2000; White, Smith, Koss, \& Figueredo, 2000; Woodin et al., 2013).

\section{Data Analysis}

Parallel factorial analyses of variance (ANOVAs) were run to analyze differences in self-objectification and objectification of the other sex as a function of participant sex and history of severe IPV victimization (IPV-V) and severe IPV perpetration (IPV-P). Assumptions of normality, homogeneity of variance, and multicollinearity were all met. Subscale data were examined post hoc to investigate whether the relationship trends 
TABLE 1. Percentage of Participants Who Reported Severe Psychological, Physical, or Sexual Intimate Partner Violence

\begin{tabular}{lcccccccc}
\hline & \multicolumn{3}{c}{ Male $(n=293)$} & & \multicolumn{3}{c}{ Female $(n=712)$} \\
\cline { 2 - 3 } & $\begin{array}{c}\text { IPV-P } \\
\text { Only }\end{array}$ & $\begin{array}{c}\text { IPV-V } \\
\text { Only }\end{array}$ & $\begin{array}{c}\text { IPV-P and } \\
\text { IPV-V }\end{array}$ & & $\begin{array}{c}\text { IPV-P } \\
\text { Only }\end{array}$ & $\begin{array}{c}\text { IPV-V } \\
\text { Only }\end{array}$ & $\begin{array}{c}\text { IPV-P and } \\
\text { IPV-V }\end{array}$ \\
\hline Psychological & 5.5 & 8.5 & 20.5 & & 9.7 & 4.6 & 16.0 \\
Physical & 2.4 & 4.8 & 13.0 & & 2.9 & 2.9 & 4.8 \\
Sexual & 0.0 & 2.4 & 9.2 & & 0.4 & 2.8 & 1.1 \\
\hline
\end{tabular}

Note. IPV-P = intimate partner violence perpetration; IPV-V = intimate partner violence victimization.

identified using the aggregate data were consistent across the three forms of IPV included in this study (severe psychological aggression, physical assault, and sexual coercion).

\section{RESULTS}

\section{Descriptive Statistics}

Prevalence rates for severe psychological, physical, and sexual IPV perpetration and victimization were calculated across participant sex and are reported in Table 1. Perpetration of more than one form of severe IPV was reported by $8.0 \%$ of participants, whereas victimization by more than one form of severe IPV was reported by $8.9 \%$ of participants. Group means for self-objectification and objectification of the other sex based on participant sex and IPV history are presented in Table 2.

TABLE 2. Mean Objectification Scores by Participant Sex and Severe Intimate Partner Violence History

\begin{tabular}{|c|c|c|c|c|c|c|c|c|c|c|}
\hline & \multicolumn{5}{|c|}{ Male } & \multicolumn{5}{|c|}{ Female } \\
\hline & \multirow[b]{2}{*}{$n$} & \multicolumn{2}{|c|}{ SOQ } & \multicolumn{2}{|c|}{ OOSQ } & \multirow[b]{2}{*}{$n$} & \multicolumn{2}{|c|}{ SOQ } & \multicolumn{2}{|c|}{ OOSQ } \\
\hline & & $M$ & $S D$ & $M$ & $S D$ & & $M$ & $S D$ & $M$ & $S D$ \\
\hline $\begin{array}{l}\text { Primary } \\
\text { perpetrator }\end{array}$ & 21 & 1.10 & 10.59 & 4.90 & 9.95 & 90 & -2.84 & 11.01 & -5.64 & 9.90 \\
\hline $\begin{array}{c}\text { Primary } \\
\text { victim }\end{array}$ & 37 & -8.46 & 12.76 & 0.08 & 10.40 & 56 & 2.39 & 10.81 & -3.93 & 10.50 \\
\hline Bidirectional & 54 & -5.23 & 10.13 & -1.89 & 10.31 & 92 & -4.80 & 11.42 & -5.02 & 9.58 \\
\hline Nonviolent & 181 & -7.12 & 10.68 & -0.22 & 9.95 & 474 & -3.11 & 11.83 & -5.28 & 10.36 \\
\hline Total & 293 & -6.36 & 11.04 & -0.12 & 10.14 & 712 & -2.86 & 11.69 & -5.19 & 10.20 \\
\hline
\end{tabular}

Note. Total scales range from -25 to 25 . Higher scores represent higher levels of objectification. SOQ = Self-Objectification Questionnaire; OOSQ = Objectification of the Other Sex Questionnaire. 


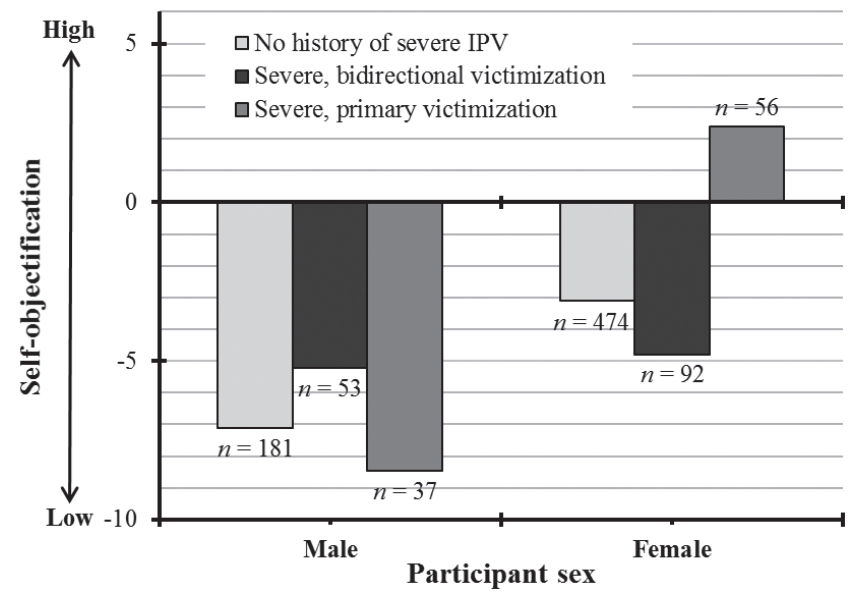

Figure 1. Self-objectification by participant sex and history of severe intimate partner violence (IPV) victimization.

\section{Self-Objectification and Intimate Partner Violence Victimization}

Self-objectification scores by participant sex and IPV-V history are displayed in Figure 1. We conducted a factorial ANOVA to assess the effects of participant sex and history of IPV-V on self-objectification. There was a significant main effect of sex on selfobjectification, $F(1,887)=21.68, p<.001, \eta^{2}=.02$. Females, as a group, scored higher $(M=-2.87, S D=11.80)$ than males $(M=-6.93, S D=10.88)$ in self-objectification, $d=.36$. There was no main effect of IPV-V on self-objectification. There was a significant interaction between sex and IPV-V, $F(2,887)=5.61, p<.01, \eta^{2}=.01$. Females with a history of primary IPV-V $(M=2.39, S D=10.81)$ demonstrated higher levels of selfobjectification than those with a history of bidirectional IPV-V $(M=-4.80, S D=11.42)$, $d=.65$, and those without a history of IPV-V $(M=-3.11, S D=11.83), d=.49$. A history of IPV-V was not predictive of self-objectification in males. These findings confirm Hypothesis 1.

\section{Objectification of the Other Sex and Intimate Partner Violence Perpetration}

Objectification of the Other sex scores by participant sex and IPV-P history are shown in Figure 2. A factorial ANOVA was computed to assess the effects of participant sex and history of IPV-P on objectification of the other sex. There was a significant main effect of sex on objectification of the other sex, $F(1,906)=35.65, p<.001, \eta^{2}=.04$. Males, as a group, scored higher $(M=-0.15, S D=10.12)$ than females $(M=-5.29, S D=10.18)$ in objectification of the other sex, $d=.51$. There was no main effect of IPV-P history on objectification of the other sex. There was a significant interaction between sex and IPV-P history, $F(2,906)=3.08, p<.05, \eta^{2}=.01$. Males with a history of primary IPV-P $(M=4.90, S D=9.95)$ demonstrated higher levels of objectification of the other sex than those with a history of bidirectional IPV-P $(M=-1.89, S D=10.31), d=.67$, and those without a history of IPV-P $(M=-0.22, S D=9.95), d=.51$. A history of IPV-P was not predictive of objectification of the other sex in females. These findings support Hypothesis 2. 


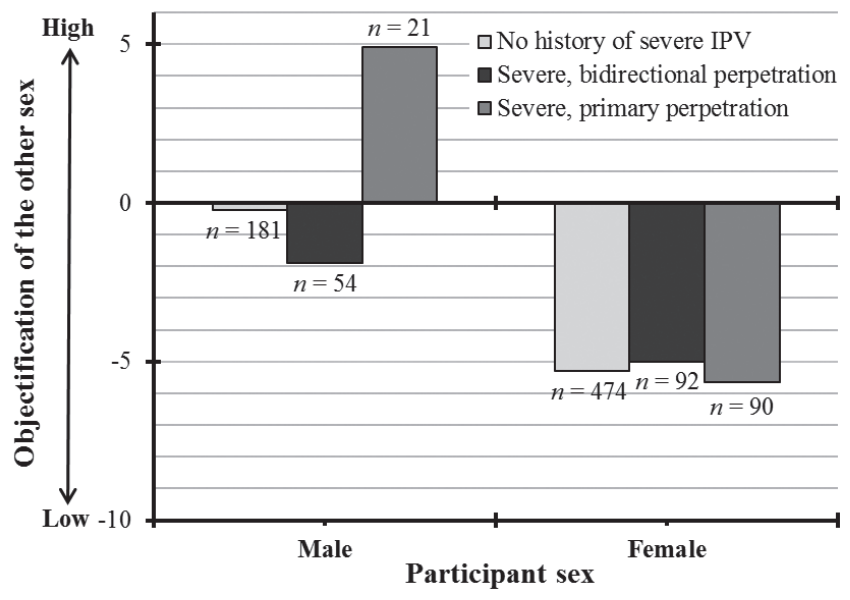

Figure 2. Objectification of the other sex by participant sex and history of severe intimate partner violence (IPV) perpetration.

\section{Revised Conflict Tactics Scale Subscales}

The reduction in power arising from the separation of the CTS2 victimization and perpetration subscales precludes reliable significance testing of separate analysis of the CTS2 subscales. However, the pattern of the relationships identified between objectification and IPV appeared to be consistent across severe psychological aggression, physical assault, and sexual coercion (Figures 3 and 4). Regarding self-objectification and history of IPV victimization, similar trends were observed across the severe CTS2 subscale data in relation to the aggregate data (see Figures 1 and 3). Although more comprehensive analyses

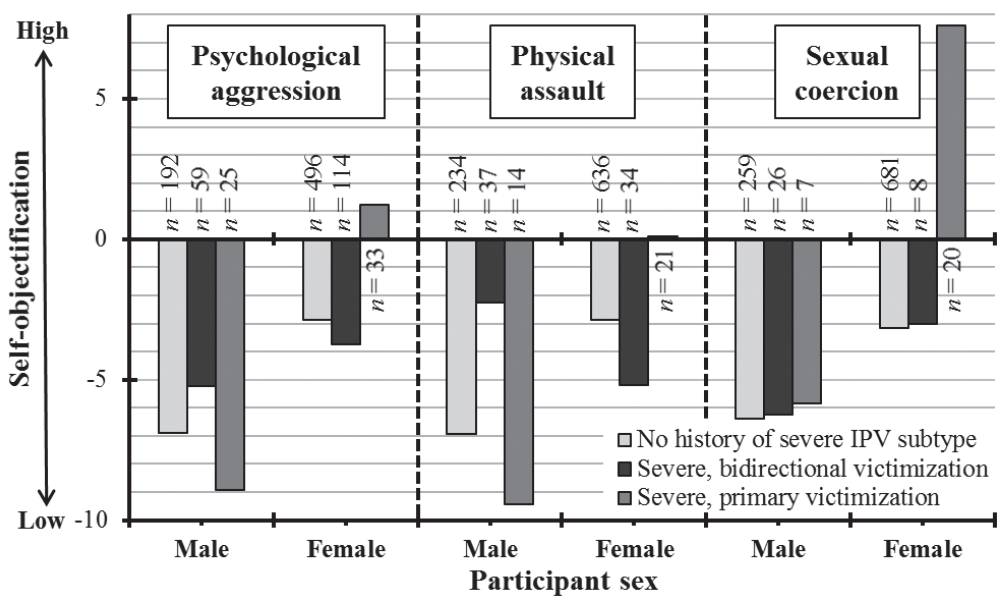

Figure 3. Self-objectification by participant sex and history of severe intimate partner violence (IPV) victimization across Revised Conflict Tactics Scale (CTS2) subscale data. Participants with "no history of severe IPV subtype" consist of those who reported no history of the specific form of IPV indicated. 


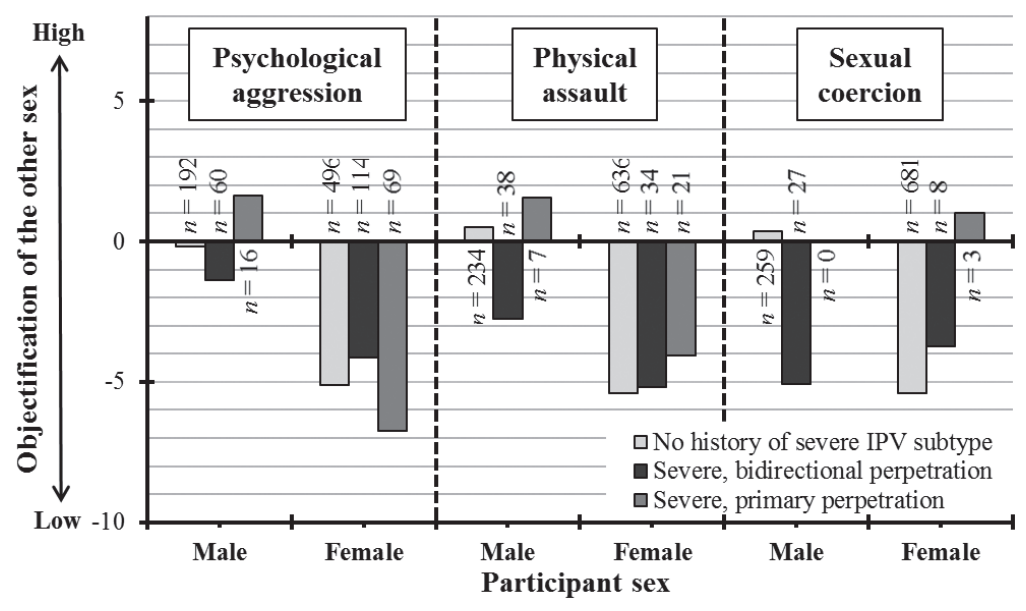

Figure 4. Objectification of the other sex by participant sex and history of severe intimate partner violence (IPV) perpetration across Revised Conflict Tactics Scale (CTS2) subscale data. Participants with "no history of severe IPV subtype" consist of those who reported no history of the specific form of IPV indicated.

are required to confirm the consistency of the current findings across discrete forms of IPV, this visual comparison of the data suggests that the aggregate CTS2 data can be considered a fair representation of the subscale data. Regarding objectification of the other sex and history of IPV perpetration, similar trends were observed across the severe CTS2 psychological aggression and physical assault subscales in relation to the aggregate data (see Figures 2 and 4). However, because very few participants reported a history of primary perpetration of sexual coercion, we draw no specific conclusions regarding this form of IPV.

\section{DISCUSSION}

This study examined whether levels of objectification of the self and other sex varied across participant sex and history of IPV. Results from this study provide support for the prior finding that men and women have distinct patterns of objectification (Strelan \& Hargreaves, 2005). Females, as a group, displayed a greater tendency to internalize an objectified perspective of themselves, and males, as a group, displayed a greater tendency to adopt an objectified perspective of the other sex. More important, sex differences were also identified in the association between self-objectification and IPV victimization and the association between objectification of the other sex and IPV perpetration.

\section{Self-Objectification and Intimate Partner Violence Victimization}

Females with a history of severe, primary IPV victimization demonstrated significantly higher levels of self-objectification than females involved in severe, bidirectional IPV and females not involved in IPV, with effect sizes for these differences falling within the moderate range ( $d=.65$ and .49 , respectively). There were no significant differences in self-objectification with respect to male IPV groups. These results confirm Hypothesis 1 
and are consistent with the prior finding that women with a history of IPV victimization are more likely to experience self-objectification than those without a history (Davidson \& Gervais, 2015). The fact that males' histories of severe, primary IPV victimization were not predictive of self-objectification suggests that gender is a consequential factor in the relationship between internalized objectification and IPV victimization. The current findings also highlight the importance of IPV directionality in the relationship between self-objectification and IPV victimization. Although a history of severe, primary IPV victimization was associated with inflated self-objectification in females, a history of severe, bidirectional IPV was not. This distinction suggests that appearance plays a greater role in the self-concepts of female IPV survivors who do not reciprocate violence against their partners than in the self-concepts of those who do reciprocate violence. Further research is needed to understand the potentially important role of IPV directionality in the relationship between self-objectification and IPV victimization in females.

\section{Objectification of the Other Sex and Intimate Partner Violence Perpetration}

Males with a history of severe, primary IPV perpetration demonstrated significantly higher levels of objectification of the other sex than males involved in severe, bidirectional IPV and males not involved in IPV, with effect sizes for these differences also falling within the moderate range ( $d=.67$ and .51 , respectively). There were no significant differences in objectification of the other sex with respect to female IPV groups. As mentioned, there were no males in the current sample who reported a history of perpetrating primary sexual coercion and, therefore, this finding can only be interpreted in relation to psychological aggression and physical assault. Nevertheless, these data support Hypothesis 2 and are consistent with the prior finding that men who commit violence against women have a greater tendency to perceive women as objects (Polaschek \& Gannon, 2004; Tilley \& Brackley, 2005). The fact that females' histories of primary IPV perpetration were not predictive of objectification of the other sex reveals that gender is also imperative in understanding the relationship between other sex objectification and IPV perpetration. Although a history of severe, primary IPV perpetration was associated with elevated levels of other sex objectification in males, a history of severe, bidirectional IPV perpetration was not. This suggests that male perpetrators of psychological and physical IPV who are not victimized by their partners place greater importance on females' appearances than those who are victimized. This reinforces the need to include measures of IPV directionality when investigating the relationship between objectification and IPV history.

\section{Implications}

The present findings indicate that males who perpetrate and females who are victimized by severe, primarily unidirectional forms of IPV hold more objectified perspectives of females than their bidirectionally violent and nonviolent counterparts. That is, they are more likely to judge females' observable traits (e.g., sex appeal) as more important than their nonobservable traits (e.g., health). These results are consistent with prior research that has found a positive relationship between IPV involvement and the endorsement of traditional gender role stereotypes, such as the stereotype that women should put the needs of others before their own (e.g., Nabors \& Jasinski, 2009).

Objectification theory posits that in cultures where women are routinely objectified, women and girls are socialized to internalize an (implicitly objectifying) observer's 
perspective of themselves (Fredrickson \& Roberts, 1997). Furthermore, although women face a common vulnerability to sexual objectification, experiences shared by particular subgroups of women can interact with experiences of objectification to produce distinct responses (Fredrickson \& Roberts, 1997). The increased levels of self-objectification that appeared in female survivors of severe, primary IPV victimization, as compared to other females, suggest that females' experiences of being victimized by IPV interact with cultural constructions of women's bodies. Thus, the relationship between gender, objectification, and IPV identified in this study may represent an important component of the individual harm caused by interdependent systems of gendered violence and oppression.

The cross-sectional design of this study precludes causal attribution. Although it is perhaps more theoretically coherent to propose that primary IPV victimization accentuates self-objectification in females, it may be the case that a tendency to internalize objectifying perspectives of women represents a risk for primary IPV victimization. Likewise, there are several potential pathways that might describe the relationship between objectification of the other sex and IPV perpetration of psychological aggression and physical assault in males. It is possible, for example, that objectifying perceptions of females may precede and contribute to psychological and physical IPV in males. Conversely, it is possible that males' propensity to objectify females is exacerbated following psychological and physical IPV perpetration as a means to increase congruence between their behaviors and attitudes. This latter explanation is consistent with longitudinal evidence demonstrating that male and female college students develop attitudes supportive of IPV, such as traditional gender role stereotypes, following involvement in violent relationships (Nabors \& Jasinski, 2009). Additional longitudinal studies are required to ascertain the direction of these relationships and further elucidate the mechanisms that underlie these associations.

Pending such examinations, the present findings have clinical implications, as understanding the relationship between IPV and objectification may help to inform intervention and prevention strategies (Davidson \& Gervais, 2015). From an intervention standpoint, these findings may prompt clinicians to screen female clients with a history of IPV victimization for experiences of internalized objectification, which may help to identify the presence of comorbid conditions associated with self-objectification and reduce their potential harm (Davidson \& Gervais, 2015). At the preventative level, media literacy programs in schools could help disseminate information to children and parents about the widespread objectification of women and girls in society. Educational programs could also provide people with tools to help them avoid internalizing objectified perceptions of themselves and others. For example, observing images that represent women as autonomous subjects and challenge traditional gender norms may make people less likely to endorse sex-role stereotypes and attitudes supportive of violence against women (Lanis \& Covell, 1995).

\section{Limitations}

The limitations of this research include the underrepresentation of male participants and the resulting imbalance in group sizes, which restricted our ability to examine the CTS2 subscales separately. In addition, psychological aggression was the predominant form of perpetration reported in our sample. Future research should more carefully investigate whether the relationships identified in this study are consistent across psychological, physical, and sexual forms of IPV, especially in relation to objectification of the other sex. In addition, although the SOQ has been used in research with both females and males, 
some items may be more suited to measuring self-objectification in females. An example noted by Calogero (2010) is strength, which represents an unobservable, competencebased trait in the SOQ. Considering the cultural emphasis on muscularity for men, strength may also qualify as an observable, appearance-based trait in the minds of males (Daniel \& Bridges, 2010). Furthermore, although the "drag and drop" method used for completing the SOQ in this study appears conceptually valid, future research should assess the validity and test-retest reliability of this novel procedure.

Another limitation of this study is that, because of the self-report nature of the SOQ and the CTS2, it is difficult to ascertain the accuracy of participants' responses. For instance, in response to the CTS2, some participants may have minimized the frequency or extent of their own violence and/or exaggerated that of their partners'. Indeed, recent research indicates discordance in the reporting of IPV perpetration between intimate partners, suggesting that men and women may experience and interpret relationship conflict differently (Currie, 1998; Renner et al., 2015). For example, research on the CTS2 indicates that, on average, women tend to minimize or discount men's use of aggression, whereas men tend to exaggerate or overstate women's violent behavior (Currie, 1998). To mitigate these issues and increase validity, future studies should supplement the SOQ and the CTS2 with additional measures (Keating, 2015; Langhinrichsen-Rohling, 2010; Woodin et al., 2013).

Finally, given that the CTS2 lacks important detail regarding the context of relationship violence, caution must be taken to not equate frequency of violent behaviors with the severity or impact of those behaviors. This is especially true when discussing bidirectional IPV because literature has identified substantial variations among bidirectionally violent couples (Langhinrichsen-Rohling, 2010). The dynamic and heterogeneous nature of bidirectional IPV may help to explain the null findings in this study concerning the relationship between bidirectional IPV victimization and self-objectification, as well as between bidirectional IPV perpetration and objectification of the other sex. As such, future research should continue to examine the potential relationship between objectification and bidirectional IPV with particular attention afforded to divergent forms of bidirectional violence.

\section{CONCLUSION}

The limitations of this study are balanced by several strengths. By including both males and females in our sample and assessing histories of both IPV perpetration and victimization, this study was able to examine cross-sex consistency and extend our currently limited knowledge of female perpetrators and males victims of IPV. Furthermore, our distinction between minor and severe incidents of IPV allowed us to focus on those most severely impacted by relationship violence, which we hope will maximize the usefulness of this study for practitioners, researchers, and decision makers. Recognizing individual similarities and differences in IPV experiences can aid in the development of more effective harm reduction strategies and help tailor the services available to those affected by IPV (Keating, 2015).

Collectively, findings from this study support the prior finding that there are gendered aspects of relationship violence that warrant attention (Hamby \& Turner, 2013) and emphasize the need to adapt IPV prevention and intervention strategies according to sex and directionality of violence. For females, experiences of severe, primary IPV victimization were associated with an increased tendency to view their bodies in observable, appearance-based terms in a manner that reflects the larger societal tendency to objectify women and their bodies. This 
indicates that female victims, in particular, may be at an increased risk for experiencing other clinically problematic conditions associated with internalized objectification, such as body shame, decreased self-esteem, depression, sexual dysfunction, and disordered eating. For males, histories of severe, primary IPV perpetration of psychological aggression and physical assault were associated with elevated levels of objectification of the other sex. This suggests that male perpetrators of psychological and physical IPV, in particular, may benefit from intervention programs that address attitudes related to the objectification of females.

In summary, our results highlight potentially important differences between males and females' cognitive correlates of IPV, despite apparent similarity in rates. As such, this research adds to literature highlighting gendered distinctions in experiences of IPV and supports the importance of taking IPV directionality into account when examining factors associated with IPV. Because IPV appears to be influenced by a complex network of factors, it is important for multiple disciplines to work together in assessing factors indicative of risk and in lessening the individual and societal burden IPV entails. By examining the relationship between IPV and objectification, this study explores a relatively new avenue of investigation in IPV research. From an exploratory perspective, this research provides insight into the cognitions of male and female perpetrators and victims of IPV and, ultimately, contributes to the goal of reducing the prevalence and costs of violence within intimate relationships.

\section{REFERENCES}

Ansara, D. L., \& Hindin, M. J. (2010). Exploring gender differences in the patterns of intimate partner violence in Canada: A latent class approach. Journal of Epidemiology and Community Health, 64, 849-854. http://dx.doi.org/10.1136/jech.2009.095208

Archer, J. (2000). Sex differences in aggression between heterosexual partners: A meta-analytic review. Psychological Bulletin, 126, 651-680.

Aubrey, J. S., Hopper, K. M., \& Mbure, W. G. (2011). Check that body! The effects of sexually objectifying music videos on college men's sexual beliefs. Journal of Broadcasting \& Electronic Media, 55, 360-379. http://dx.doi.org/10.1080/08838151.2011.597469

Calogero, R. M. (2011). Operationalizing self-objectification: Assessment and related methodological issues. In R. M. Calogero, S. Tantleff-Dunn, \& J. K. Thompson (Eds.), Self-objectification in women: Causes, consequences, and counteractions (pp. 23-49). Washington, DC: American Psychological Association.

Centers for Disease Control and Prevention. (2010). Intimate partner violence: Consequences. Washington DC: Author. Retrieved from http://www.cdc.gov/violenceprevention/intimate partnerviolence/consequences.html

Currie, D. H. (1998). Violent men or violent women? Whose definition counts? In R. K. Bergen (Ed.), Issues in intimate violence (pp. 97-111). Thousand Oaks, CA: Sage.

Dakanalis, A., Timko, C. A., Clerici, M., Zanetti, M. A., \& Riva, G. (2014). Comprehensive examination of the trans-diagnostic cognitive behavioral model of eating disorders in males. Eating Behaviors, 15, 63-67. http://dx.doi.org/10.1016/j.eatbeh.2013.10.003

Daniel, S., \& Bridges, S. K. (2010). The drive for muscularity in men: Media influences and objectification theory. Body Image, 7, 32-38. http://dx.doi.org/10.1016/j.bodyim.2009.08.003

Davidson, M. M., \& Gervais, S. J. (2015). Violence against women through the lens of objectification theory. Violence Against Women, 21, 330-354. http://dx.doi.org/10.1177/1077801214568031

Davidson, M. M., Gervais, S. J., Canivez, G. L., \& Cole, B. P. (2013). A psychometric examination of the Interpersonal Sexual Objectification Scale among college men. Journal of Counseling Psychology, 60, 239-250. http://dx.doi.org/10.1037/a0032075 
Davidson, M. M., Gervais, S. J., \& Sherd, L. W. (2015). The ripple effects of stranger harassment on objectification of self and others. Psychology of Women Quarterly, 39, 53-66. http://dx.doi .org/10.1177/0361684313514371

Fass, D. F., Benson, R. I., \& Leggett, D. G. (2008). Assessing prevalence and awareness of violent behaviors in the intimate partner relationships of college students using Internet sampling. Journal of College Student Psychotherapy, 22(4), 66-75. http://dx.doi.org/ $10.1080 / 87568220801952248$

Fredrickson, B. L., \& Roberts, T. (1997). Objectification theory: Toward understanding women's lived experiences and mental health risks. Psychology of Women Quarterly, 21, 173-206. http://dx.doi.org/10.1111/j.1471-6402.1997.tb00108.x

Fredrickson, B. L., Roberts, T., Noll, S. M., Quinn, D. M., \& Twenge, J. M. (1998). That swimsuit becomes you: Sex differences in self-objectification, restrained eating, and math performance. Journal of Personality and Social Psychology, 75, 269-284. http://dx.doi.org/10.1037/00223514.75.1.269

Frieze, I. H. (2000). Violence in close relationships-development of a research area: Comment on Archer (2000). Psychological Bulletin, 126, 681-684.

Gervais, S. J., \& Davidson, M. M. (2013). Objectification among college women in the context of intimate partner violence. Violence and Victims, 28, 36-49. Retrieved from http://www.springerpub .com/violence-and-victims.html

Gervais, S. J., DiLillo, D., \& McChargue, D. (2014). Understanding the link between men's alcohol use and sexual violence perpetration: The mediating role of sexual objectification. Psychology of Violence, 4, 156-169. http://dx.doi.org/10.1037/a0033840

Graham-Kevan, N., \& Archer, J. (2003). Intimate terrorism and common couple violence: A test of Johnson's predictions in four British samples. Journal of Interpersonal Violence, 18, 1247-1270. http://dx.doi.org/10.1177/0886260503256656

Green, M. A., Ohrt, T. K., Nguyen, C., Blasko, K., Khatiwada, S., Martin, A., . . Marie, C. (2014). Heart rate and affective reactions to state self-objectification as a function of gender. Basic and Applied Social Psychology, 36, 259-271. http://dx.doi.org/10.1080/01973533.2014.900620

Hald, G. M., Malamuth, N., \& Yuen, C. (2010). Pornography and attitudes supporting violence against women: Revisiting the relationships in nonexperimental studies. Aggressive Behavior, 36, 14-20. http://dx.doi.org/10.1002=ab.20328

Hamby, S. (2016). Self-report measures that do not produce gender parity in intimate partner violence: A multi-study investigation. Psychology of Violence, 6, 323-335. http://dx.doi .org/10.1037/a0038207

Hamby, S., \& Turner, H. (2013). Measuring teen dating violence in males and females: Insights from the National Survey of Children's Exposure to Violence. Psychology of Violence, 3, 323-339. http://dx.doi.org/10.1037/a0029706

Heimerdinger-Edwards, S. R., Vogel, D. L., \& Hammer, J. H. (2011). Extending sexual objectification theory and research to minority populations, couples, and men. The Counseling Psychologist, 39, 140-152. http://dx.doi.org/10.1177/0011000010383894

Henning, K., Renauer, B., \& Holdford, R. (2006). Victim or offender? Heterogeneity among women arrested for intimate partner violence. Journal of Family Violence, 21, 351-368. http://dx.doi .org/10.1007/s10896-006-9032-4

Hill, M. S., \& Fischer, A. R. (2008). Examining objectification theory: Lesbian and heterosexual women's experiences with sexual and self-objectification. The Counseling Psychologist, 36, 745-776. http://dx.doi.org/10.1177/0011000007301669

Johnson, M. P. (2006). Conflict and control: Gender symmetry and asymmetry in domestic violence. Violence Against Women, 12, 1003-1018. http://dx.doi.org/10.1177/1077801206293328

Keating, B. (2015). Violence against women: A disciplinary debate and challenge. The Sociological Quarterly, 56, 108-124. http://dx.doi.org/10.1111/tsq.12075

Langhinrichsen-Rohling, J. (2010). Controversies involving gender and intimate partner violence in the United States. Sex Roles, 62, 179-193. http://dx.doi.org/10.1007/s11199-009-9628-2 
Lanis, K., \& Covell, K. (1995). Images of women in advertisements: Effects on attitudes related to sexual aggression. Sex Roles, 32, 639-649. http://dx.doi.org/10.1007/BF01544216

Lehrner, A., \& Allen, N. E. (2014). Construct validity of the Conflict Tactics Scales: A mixed-method investigation of women's intimate partner violence. Psychology of Violence, 4, 477-490. http://dx.doi.org/10.1037/a0037404

Lindberg, S. M., Hyde, J. S., \& McKinley, N. M. (2006). A measure of objectified body consciousness for preadolescent and adolescent youth. Psychology of Women Quarterly, 30, 65-76. http://dx.doi.org/10.1111/j.1471-6402.2006.00263.x

Loughnan, S., \& Pacilli, M. G. (2014). Seeing (and treating) others as sexual objects: Toward a more complete mapping of sexual objectification. TPM, 21, 309-325. Retrieved from http://www .tpmap.org/wp-content/uploads/2015/11/21.3.6.pdf

Martins, Y., Tiggemann, M., \& Kirkbride, A. (2007). Those speedos become them: The role of selfobjectification in gay and heterosexual men's body image. Personality \& Social Psychology Bulletin, 33, 634-647. http://dx.doi.org/10.1177/0146167206297403

Melton, H. C., \& Sillito, C. L. (2012). The role of gender in officially reported intimate partner abuse. Journal of Interpersonal Violence, 27, 1090-1111.http://dx.doi.org/10.1177/0886260511424498

Mercurio, A. E., \& Landry, L. J. (2008). Self-objectification and well-being: The impact of selfobjectification on women's overall sense of self-worth and life satisfaction. Sex Roles, 58, 458-466. http://dx.doi.org/10.1007/s11199-007-9357-3

Moradi, B., \& Huang, Y. (2008). Objectification theory and psychology of women: A decade of advances and future directions. Psychology of Women Quarterly, 32, 377-398. http://dx.doi .org/10.1111/j.1471-6402.2008.00452.x

Nabors, E., \& Jasinski, J. (2009). Intimate partner violence perpetration among college students: The role of gender role and gendered violence attitudes. Feminist Criminology, 4(1), 57-82. http://dx.doi.org/10.1177/1557085108325235

Noll, S. M., \& Fredrickson, B. L. (1998). A mediational model linking self-objectification, body shame, and disordered eating. Psychology of Women Quarterly, 22, 623-636. http://dx.doi .org/10.1111/j.1471-6402.1998.tb00181.x

O'Leary, K. D. (2000). Are women really more aggressive than men in intimate relationships? Comment on Archer (2000). Psychological Bulletin, 126, 685-689.

Polaschek, D. L. L., \& Gannon, T. A. (2004). The implicit theories of rapists: What convicted offenders tell us. Sexual Abuse, 16, 299-314. http://dx.doi.org/10.1177/107906320401600404

Renner, L. M., Reese, L. S., Peek-Asa, C., \& Ramirez, M. (2015). Reporting patterns of unidirectional and bidirectional verbal aggression and physical violence among rural couples. Journal of Family Violence, 30, 1069-1078. http://dx.doi.org/10.1007/s10896-015-9737-3

Riva, G., Gaudio, S., \& Dakanalis, A. (2015). The neuropsychology of self-objectification. European Psychologist, 20, 34-43. http://dx.doi.org/10.1027/1016-9040/a000190

Statistics Canada. (2011). Family violence in Canada: A statistical profile. Retrieved from http:// www.statcan.gc.ca/pub/85-002-x/2013001/article/11805/11805-3-eng.htm

Straus, M. A. (2004). Prevalence of violence against dating partners by male and female university students worldwide. Violence Against Women, 10, 790-811. http://dx.doi.org/10.1177/ 1077801204265552

Straus, M. A. (2006). Future research on gender symmetry in physical assaults on partners. Violence Against Women, 12, 1086-1097. http://dx.doi.org/10.1177/1077801206293335

Straus, M. A. (2008). Dominance and symmetry in partner violence by male and female university students in 32 nations. Children and Youth Services Review, 30, 252-275. http://dx.doi .org/10.1016/j.childyouth.2007.10.004

Straus, M. A., Hamby, S. L., Boney-McCoy, S., \& Sugarman, D. B. (1996). The Revised Conflict Tactics Scale (CTS2): Development and preliminary psychometric data. Journal of Family Issues, 17, 283-316. http://dx.doi.org/10.1177/019251396017003001

Strelan, P., \& Hargreaves, D. (2005). Women who objectify other women: The vicious circle of objectification? Sex Roles, 52, 707-712. http://dx.doi.org/10.1007/s11199-005-3737-3 
Tiggemann, M., \& Lynch, J. E. (2001). Body image across the life span in adult women: The role of self-objectification. Developmental Psychology, 37, 243-253. http://dx.doi.org/10.1037/00121649.37.2.243

Tilley, D. S., \& Brackley, M. (2005). Men who batter intimate partners: A grounded theory study of the development of male violence in intimate partner relationships. Issues in Mental Health Nursing, 26, 281-297. http://dx.doi.org/10.1080/01612840590915676

Tjaden, P., \& Thoennes, N. (2000). Prevalence and consequences of male-to-female and female-tomale intimate partner violence as measured by the National Violence Against Women Survey. Violence Against Women, 6, 142-161. http://dx.doi.org/10.1177/10778010022181769

Tully, K. E. (2012). Objectification of women and intimate partner violence in homosocial groups (Doctoral dissertation). Retrieved from ProQuest Dissertations and Theses database. (1032561776)

Vega, E. M., \& O'Leary, K. D. (2007). Test-retest reliability of the Revised Conflict Tactics Scales (CTS2). Journal of Family Violence, 22, 703-708. http://dx.doi.org/10.1007/s10896-007-9118-7

Walsh, Z., Swogger, M. T., O'Connor, B. P., Chatav Schonbrun, Y., Shea, M. T., \& Stuart, G. L. (2010). Subtypes of partner violence perpetrators among male and female psychiatric patients. Journal of Abnormal Psychology, 119, 563-574. http://dx.doi.org/10.1037/a0019858

Ward, L. M., Vandenbosch, L., \& Eggermont, S. (2015). The impact of men's magazines on adolescent boys' objectification and courtship beliefs. Journal of Adolescence, 39, 49-58. http://dx.doi.org/10.1016/j.adolescence.2014.12.004

White, J. W., Smith, P. H., Koss, M. P., \& Figueredo, A. J. (2000). Intimate partner aggression-what have we learned? Comment on Archer (2000). Psychological Bulletin, 126, 690-696.

Wiseman, M. C., \& Moradi, B. (2010). Body image and eating disorder symptoms in sexual minority men: A test and extension of objectification theory. Journal of Counseling Psychology, 57, 154-166. http://dx.doi.org/10.1037/a0018937

Woodin, E. M., Sotskova, A., \& O'Leary, K. D. (2013). Intimate partner violence assessment in an historical context: Divergent approaches and opportunities for progress. Sex Roles, 69, 120-130. http://dx.doi.org/10.1007/s11199-013-0294-z

World Health Organization. (2012). Understanding and addressing violence against women: Intimate partner violence. Geneva, Switzerland: Author.

Acknowledgments. This research was supported in part by a grant from the Irving K. Barbour Endowment Fund and by the Social Sciences and Humanities Research Council of Canada.

Correspondence regarding this article should be directed to Zach Walsh, $\mathrm{PhD}$, University of British Columbia, Department of Psychology, 3333 University Way, Kelowna BC, V1V 1V7. E-mail: zachary.walsh@ubc.ca 